Research Paper: Immunology

\title{
A fragment of the alarmin prothymosin a as a novel biomarker in murine models of bacteria-induced sepsis
}

\author{
Pinelopi Samara ${ }^{1}$, Vivi Miriagou ${ }^{2}$, Michael Zachariadis ${ }^{1,3}$, Olga Mavrofrydi ${ }^{1}$, Vasilis

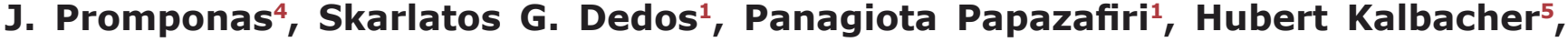 \\ Wolfgang Voelter ${ }^{5}$ and Ourania Tsitsilonis ${ }^{1}$ \\ ${ }^{1}$ Department of Biology, National and Kapodistrian University of Athens, Athens, Greece \\ ${ }^{2}$ Laboratory of Bacteriology, Hellenic Pasteur Institute, Athens, Greece \\ 3 Institute of Biosciences and Applications, National Center for Scientific Research "Demokritos", Agia Paraskevi, Athens, \\ Greece \\ ${ }^{4}$ Bioinformatics Research Laboratory, Department of Biological Sciences, University of Cyprus, Nicosia, Cyprus \\ ${ }^{5}$ Interfaculty Institute of Biochemistry, University of Tübingen, Tübingen, Germany \\ Correspondence to: Ourania Tsitsilonis, email: rtsitsil@biol.voa.gr \\ Pinelopi Samara, email: psamara@biol.voa.gr
}

Keywords: apoptosis, biomarker, Klebsiella pneumoniae, proTa(100-109), sepsis, Immunology and Microbiology Section, Immune response, Immunity

Received: December 15, $2016 \quad$ Accepted: April 17, $2017 \quad$ Published: May 24, 2017

Copyright: Samara et al. This is an open-access article distributed under the terms of the Creative Commons Attribution License 3.0 (CC BY 3.0), which permits unrestricted use, distribution, and reproduction in any medium, provided the original author and source are credited.

\section{ABSTRACT}

Sepsis is a life-threatening condition that requires urgent care. Thus, the identification of specific and sensitive biomarkers for its early diagnosis and management are of clinical importance. The alarmin prothymosin alpha (proTa) and its decapeptide proTa(100-109) are immunostimulatory peptides related to cell death. In this study, we generated bacterial models of sepsis in mice using two Klebsiella pneumoniae strains (L-78 and ATCC 43816) and monitored sepsis progression using proTa(100-109) as a biomarker. Serum concentration of proTa(100-109) gradually increased as sepsis progressed in mice infected with L-78, a strain which, unlike ATCC 43816, was phagocytosed by monocytes/macrophages. Analysis of splenocytes from L-78-infected animals revealed that post-infection spleen monocytes/macrophages were gradually driven to caspase-3-mediated apoptosis. These results were verified in vitro in L-78-infected human monocytes/macrophages. Efficient phagocytosis of L-78 by monocytes stimulated their apoptosis and the concentration of proTa(100-109) in culture supernatants increased. Human macrophages strongly phagocytosed L-78, but resisted cell death. This is the first report suggesting that high levels of proTa(100-109) correlate, both in vitro and in vivo, with increased percentages of cell apoptosis. Moreover, we showed that low levels of proTa(100-109) early postinfection likely correlate with sepsis resolution and thus, the decapeptide could eventually serve as an early surrogate biomarker for predicting bacteria-induced sepsis outcome.

\section{INTRODUCTION}

Sepsis (from Greek $\sigma \tilde{\eta} \psi 1 \varsigma$, decay) is a lifethreatening response to infection, leading to excessive tissue damage, organ failure, and death. It is associated with factors related to the invading pathogens, most commonly Gram-negative bacteria, as well as the status of the host's immune system [1]. Excessive immune cell activation leads to release of inflammatory mediators, determined usually in blood samples and used as biomarkers of infection. 
Established sepsis biomarkers include cytokines (e.g. interleukin 6 and tumor necrosis factor alpha $[\mathrm{TNF}-\alpha]$ ), lactate, the acute-phase C-reactive protein, the pro-hormone procalcitonin and damage-associated molecular patterns (DAMPs)/cell surface receptors (e.g. triggering receptor expressed on myeloid cells-1) [2]. Among DAMPs, the prototype alarmin high-mobility group box 1 protein (HMGB1) has been extensively studied both as a biomarker and a therapeutic target [3]. Nevertheless, sepsis diagnosis and assessment of its severity is rather complex because of its heterogeneity and the lack of sensitive and specific assays discriminating infectious from non-infectious cases. Consequently, novel surrogate biomarkers are needed.

Experimental animal studies are essential in identifying and characterizing the pathophysiological stages of sepsis and for developing new therapeutic strategies and diagnostic tools. Bacterial infusion in mice is the most widely used model, as introduction of a single pathogen under controlled conditions provides reproducibility of the infection [4].

Our research group studies the immune-related mechanisms of action of the polypeptide prothymosin alpha (proT $\alpha)$ and its immunologically active fragment proT $\alpha(100-109)$ [5]. ProT $\alpha(100-109)$ is generated upon truncation of proT $\alpha$ by activated caspase- 3 , in cells undergoing apoptosis [6]. Both molecules extracellularly act as alarmins, ligate TLR-4, induce the maturation of dendritic cells, and mount $\mathrm{T}_{\mathrm{H}} 1$-biased immune responses in vitro [7-9]. We hypothesized that if proTa and proTa(100-109) are released from damaged cells, they could serve as immune stimuli, acting similarly to HMGB1 [10]. To detect and quantify the extracellular release of these peptides, we previously established a highly sensitive ELISA for proT $\alpha(100-109)$ and induced massive cell death in mice, by infecting them with Streptococcus pyogenes (S. pyogenes). We showed that the concentration of proT $\alpha(100-109)$ in serum of $S$. pyogenes-infected mice was significantly increased during the progress of infection [11].

To further evaluate the role of proT $\alpha(100-109)$ as a sepsis biomarker, we used Klebsiella pneumoniae ( $K$. pneumoniae) as challenge-microorganism, a Gram-negative bacterium associated with aggressive infections (e.g. septicemia and pneumonia) and numerous nosocomial outbreaks [12]. We selected two $K$. pneumoniae strains of diverse virulence and properties [13]. We analyzed $K$. pneumoniae infection in mice and in human cells in vitro, focusing on the mechanisms via which these bacteria kill innate immune cells and lead to the generation of proT $\alpha(100-109)$. We further validated our results in a "moderate" model of sepsis in mice, and showed that early post-infection (pi) proTa(100-109) serum levels can predict mortality of individual mice infected with $K$. pneumoniae.

\section{RESULTS}

\section{Low serum levels of proT $\alpha(100-109)$ were detected early post-infection with L-78}

Animals were infected with the clinically isolated strain L-78, which is of low virulence $(50 \%$ lethal dose $\left[\mathrm{LD}_{50}\right]>10^{8}$ colony-forming units [CFU]), highly resistant to antibiotics and endocytosed by monocytes/ macrophages, and with the ATCC 43816 strain, which is highly infective $\left(\mathrm{LD}_{50}=5 \times 10^{3} \mathrm{CFU}\right)$, sensitive to antibiotics and not endocytosed. Based on the Kaplan-Meier survival curves of Tzouvelekis [13] for immunocompetent mice, animals were intraperitoneally (ip) infected with $10^{8} \mathrm{CFU}$ of L-78, and $5 \times 10^{3}$ or $10^{6}$ CFU of ATCC 43816. Blood samples were collected at the time points shown in Figure 1. Mouse sera were analyzed by our ELISA for proT $\alpha(100-109)$ [11]. To rule out obvious non-specific interactions between proT $\alpha(100-$ 109)-specific Abs and any cross-reacting substance present in murine serum, we assessed if the primary structure of the decapeptide can be depicted within the amino acid sequence of currently known mouse proteins or proteins known to be encoded in K. pneumoniae (Supplementary Table S1). A scan with the regular expression pattern against the proteins encoded in the most current version of the mouse genome showed that cross-reactivity was unlikely to occur.

In animals infected with $10^{8} \mathrm{CFU}$ of L-78, serum concentration of proT $\alpha(100-109)$ gradually increased during the course of infection with maximum quantity $(5.72 \mathrm{ng} / \mathrm{mL})$ detected at $48 \mathrm{~h}$ pi (Figure 1A), which further reduced to background levels at $96 \mathrm{~h}$ (data not shown). Mice infected with $10^{6} \mathrm{CFU}$ of ATCC 43816 exhibited a sharp increase in serum concentration of proT $\alpha(100-109)$ already in the first three hours pi $(4.53 \mathrm{ng} /$ $\mathrm{mL})$ which remained relatively constant up to $15 \mathrm{~h}$ pi (6.29 $\mathrm{ng} / \mathrm{mL}$ ) (Figure 1B). The distinct patterns of rise in the levels of proT $\alpha(100-109)$ among the two murine groups could be attributed to the diverse virulence of the strains. Specifically, animals injected with L-78 showed transient signs of infection and all resisted infection and recovered within $48 \mathrm{~h}$, while animals administered $10^{6} \mathrm{CFU}$ of ATCC 43816 died at $15 \mathrm{~h}$ pi, due to generalized sepsis. Mice infected with $5 \times 10^{3}$ CFU of ATCC 43816 ("moderate" model of sepsis), showed a different pattern of increase in serum proT $\alpha(100-109)$ levels, with some animals showing high and others low concentrations of the decapeptide early pi (Figure 1C). This group of mice was used to validate our results and data are further analyzed and discussed. To evaluate the impact of traumatic injections on $\operatorname{proT} \alpha(100-109)$ levels, we additionally collected serum from mice punctured ip and injected with saline. No differences in proT $\alpha(100-109)$ levels prior and $3 \mathrm{~h}$ after traumatic injection were observed (data not shown). 

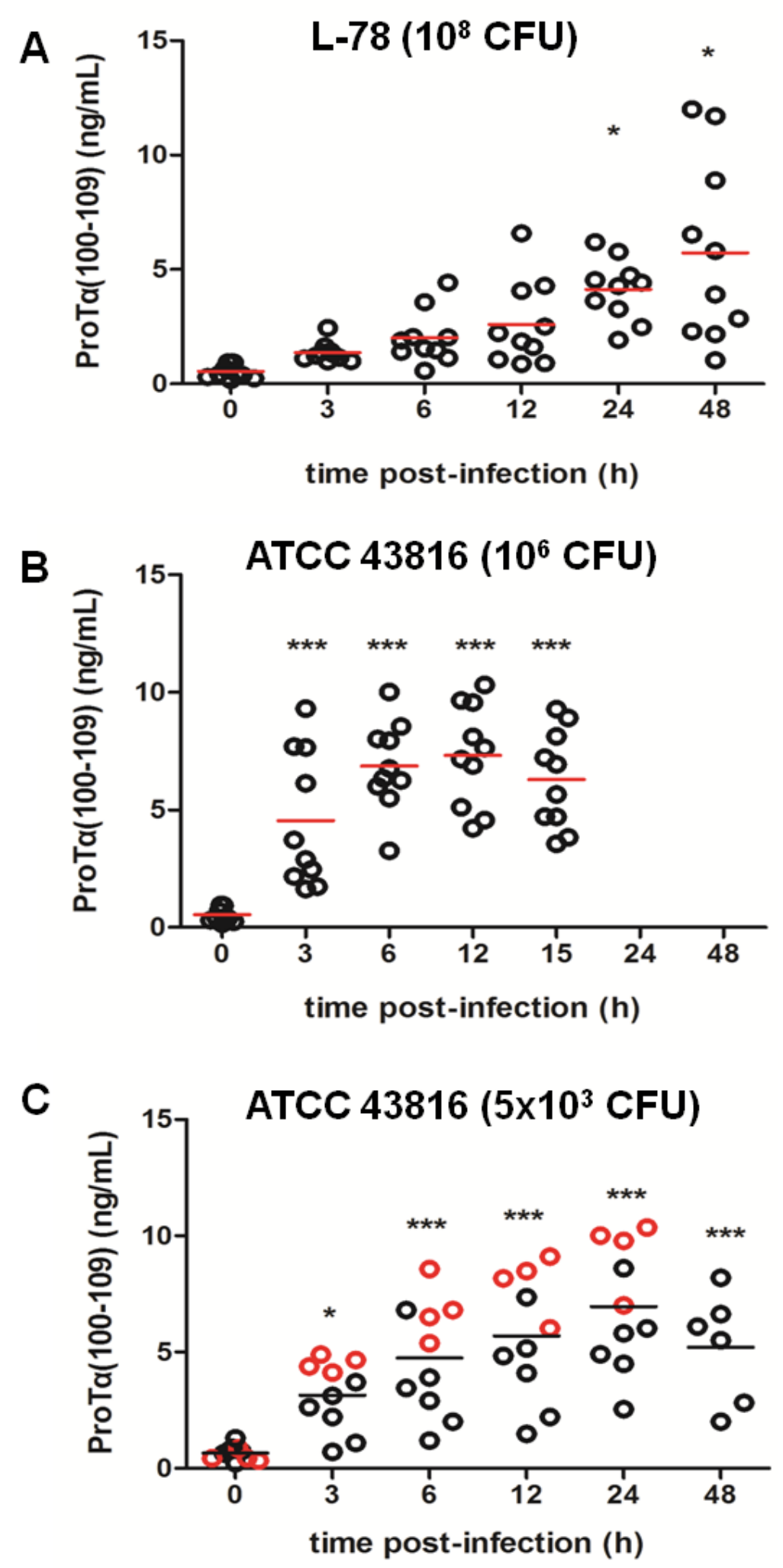

Figure 1: Concentration of proTa(100-109) in serum of mice during Klebsiella pneumoniae infection. CD-1 mice were intraperitoneally infected with L-78 A. and ATCC 43816 B., C., respectively. Serum samples were collected prior to infection (0 h), at 3, 6, 12, 24, and $48 \mathrm{~h}$ post-infection (pi) for the L-78 and the $5 \times 10^{3}$ CFU ATCC 43816 groups A. and $\mathbf{C}$., and at 3, 6, 12, and $15 \mathrm{~h}$ pi for the $10^{6}$ CFU ATCC 43816 group B.. Each circle represents the concentration of proT $\alpha(100-109)$ from a single mouse at the corresponding time point. In $\mathrm{C}$, red cirles mark mice that died between $30-45 \mathrm{~h}$ pi. Horizontal bars indicate mean values. ${ }^{*}, p<0.05 ; * * *, p<0.001$ after oneway ANOVA followed by Dunnett's test (vs $0 \mathrm{~h}$ ). 
A

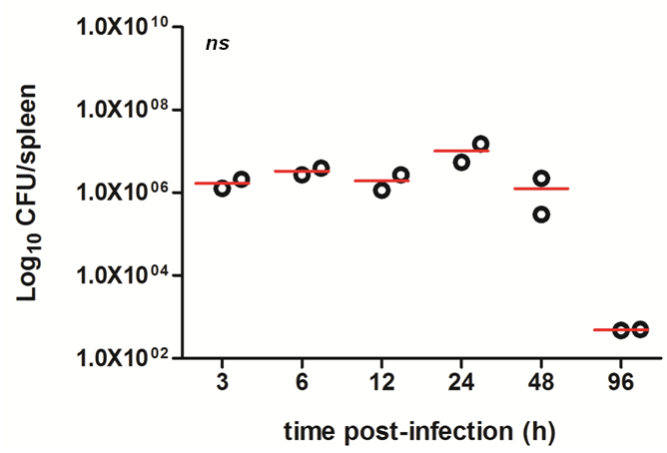

B

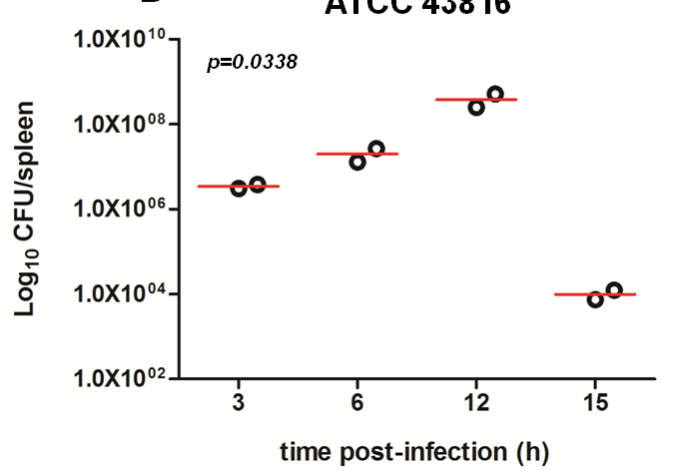

Figure 2: Bacterial load in the spleen of CD-1 mice infected with $10^{8}$ CFU L-78 A. and $10^{6}$ CFU ATCC 43816 B. Two mice from each group were sacrificed at the same time points as in Figures 1A and 1B and for an additional time point (96 h) for L-78 infected animals to confirm bacterial clearance. Spleen homogenates were plated on agar and CFU counts were determined. Data are Log ${ }_{10} \mathrm{CFU} /$ spleen from two independent experiments. ns, not significant after one-way ANOVA.

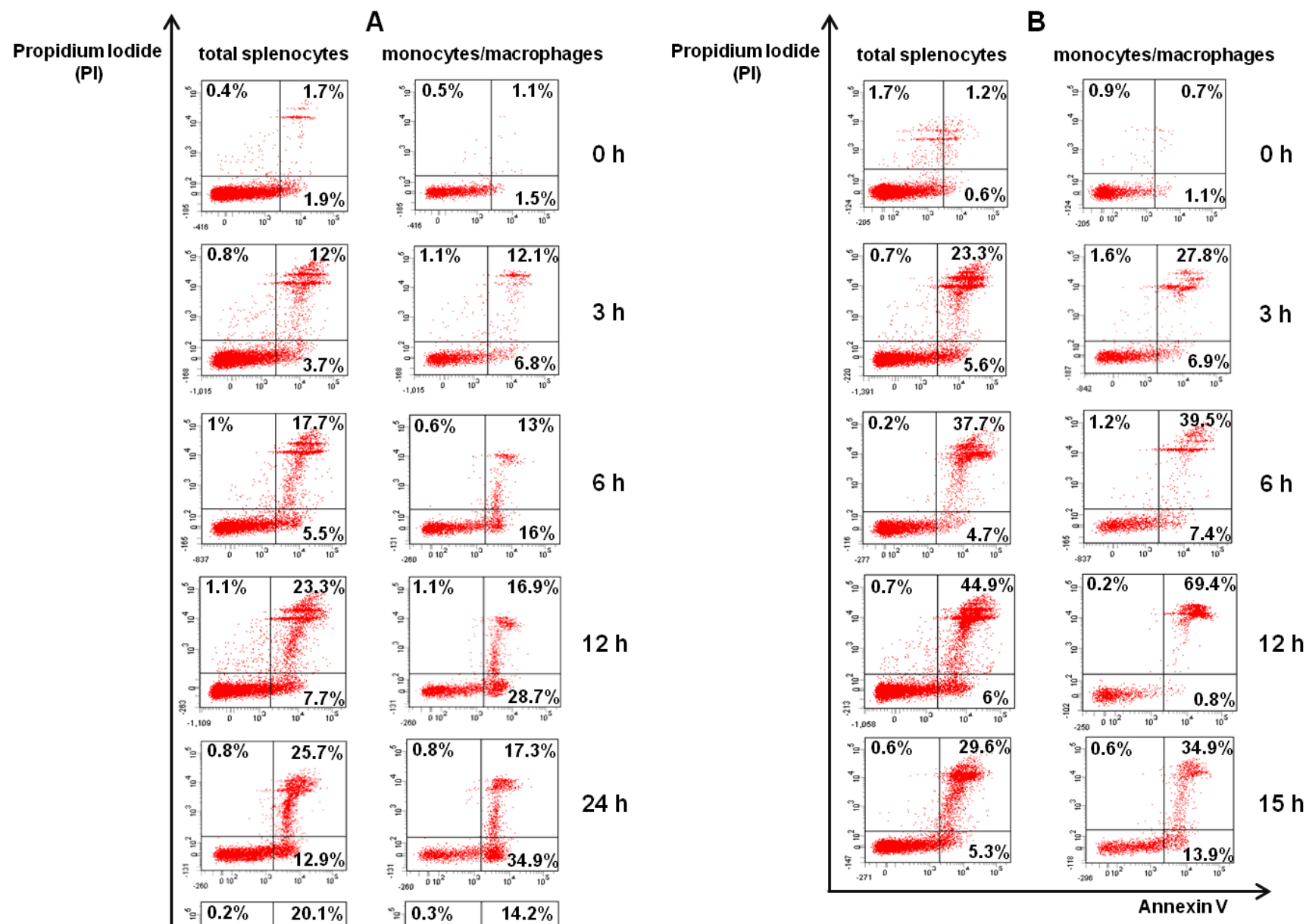

$48 \mathrm{~h}$

Figure 3: Spleen monocytes/macrophages infected with L-78 were driven to apoptosis. Total splenocytes and spleen monocytes/macrophages isolated from $K$. pneumoniae infected-mice at the indicated time points post-infection $(0-48 \mathrm{~h}$ for L-78 A. and 0-15 h for ATCC 43816 B.) were stained with Annexin V/PI and analyzed by flow cytometry. Percentages in lower right quadrants are apoptotic cells (Annexin V+PI-), and in upper quadrants necrotic cells (PI+). Dot plots shown are from one representative animal out of two independently analyzed with similar results. 
Spleen bacterial load increased in ATCC 43816-infected mice

At the same time points to blood collection, spleens from two mice of the L-78 and $10^{6}$ CFU ATCC 43816 groups were removed and bacterial load, expressed in $\mathrm{CFU}$, was determined. Spleens were not contaminated with intestinal or other external bacteria during isolation, and the individual colonies on the plates were typical for Klebsiella. We sacrificed only two mice per time point per model based on the 3R principle (Replacement, Reduction, Refinement) for laboratory animal use, to verify similar results available in our laboratory from previous experiments.

It is well documented that ip administration of bacteria leads to rapid and effective delivery to the bloodstream resulting in systemic infection [14]. In animals infected with $10^{8} \mathrm{CFU}$ of L-78 $(0 \mathrm{~h})$, the number of bacteria that entered the spleen between 3-12 h was approximately $10^{6} \mathrm{CFU}$, increased to $\sim 10^{7} \mathrm{CFU}$ at 24 $\mathrm{h}$ and decreased thereafter (Figure 2A). In contrast, in animals that were infected with $10^{6} \mathrm{CFU}$ of ATCC 43816, the bacterial load in spleen significantly increased between
6-12 h (up to $\sim 7 \times 10^{8} \mathrm{CFU}$ at $12 \mathrm{~h}$ ) and sharply decreased afterward, as animals died due to generalized sepsis at $15 \mathrm{~h}$ ( $p=0.0338$; Figure 2B). Apparently, ATCC 43816, although injected at a lower inoculum, multiplied rapidly in vivo, and led to multi-organ failure within $15 \mathrm{~h}$ pi. At this time point, generalized sepsis was accompanied by extensive necrosis of vital internal organs (including spleen), and lower bacterial spleen load was detected $\left(\sim 10^{4}\right.$ at $\left.15 \mathrm{~h}\right)$.

\section{L-78 infection induced apoptosis and ATCC 43816 necrosis in mouse spleen cells}

The type and extent of cell death in spleen cells were evaluated by flow cytometry, using Annexin V and propidium iodide (PI) staining. We analyzed total splenocytes, as well as spleen monocytes/macrophages $(\mathrm{CD} 11 \mathrm{~b}+)$ (Supplementary Figure $\mathrm{S} 1)$. As shown in Figure 3, prior to infection $(0 \mathrm{~h})$, few mouse splenocytes were apoptotic $(\sim 2 \%$ Annexin V+PI-). During the course of L-78 infection (3-48 h), percentages of apoptotic splenocytes gradually increased (up to $12.9 \%$ ) (Figure 3A, left panel). This was better shown in spleen

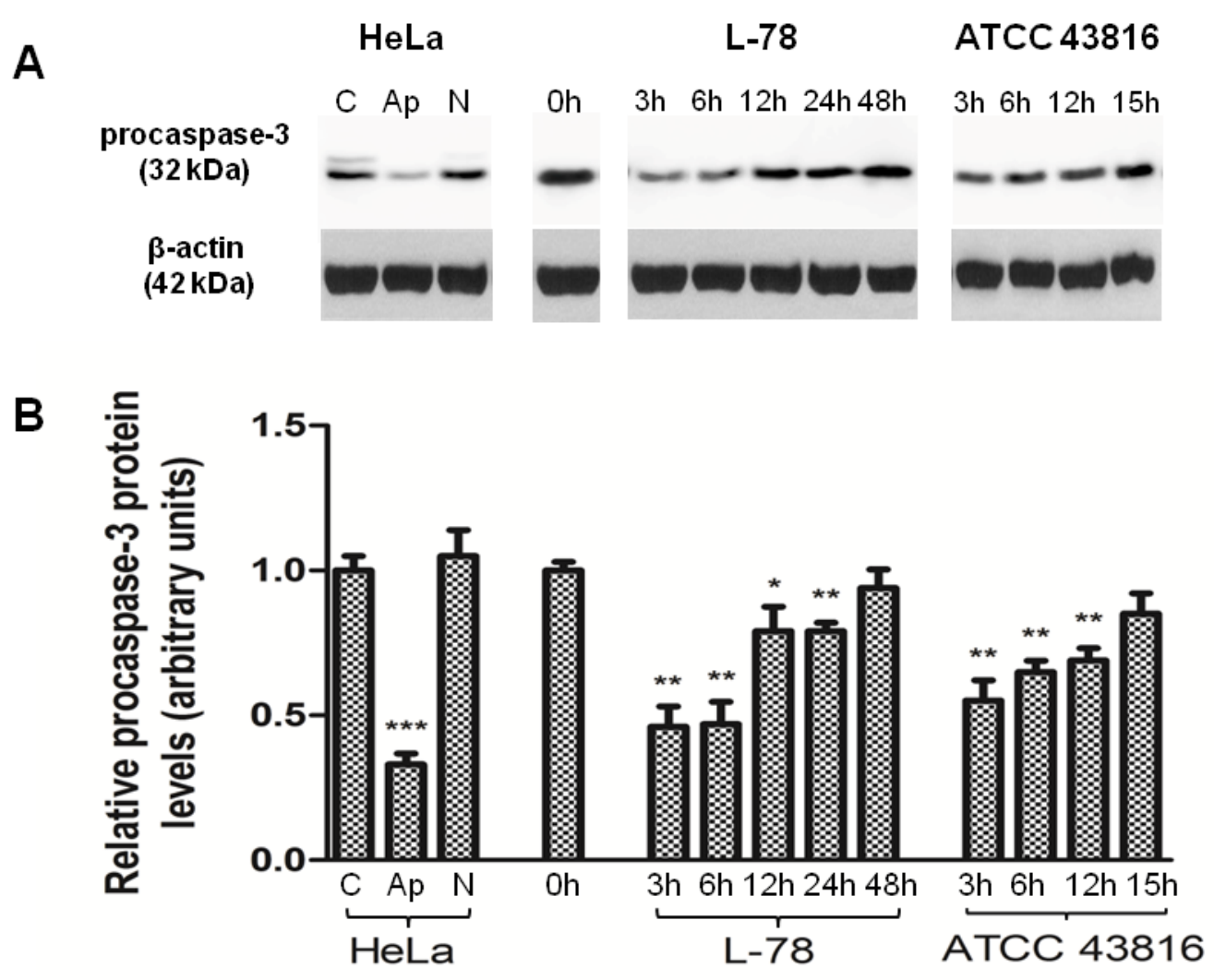

Figure 4: Procaspase-3 levels decreased early after L-78 infection in murine spleen lysates. A. Total protein extracts of pooled splenocytes from L-78- and ATCC 43816-infected mice were immunoblotted against procaspase-3. Apoptotic and necrotic HeLa cells were used as controls. $\beta$-Actin was used as a loading control. All samples were run on and cut from the same gel. B. Densitometric analysis of procaspase-3. Data are means \pm SD from three independent experiments. C, HeLa cells incubated in medium; Ap, apoptotic HeLa cells; N, necrotic HeLa cells. *, $p<0.05 ; * *, p<0.01$; **, $p<0.001$, compared with control after Student's unpaired $t$-test. 
monocytes/macrophages, where percentages of apoptotic cells significantly increased up to $24 \mathrm{~h}(34.9 \%)$ and then decreased (Figure 3A, right panel). In animals infected with $10^{6} \mathrm{CFU}$ of ATCC 43816, the percentages of apoptotic total splenocytes were low and remained unaltered (Figure 3B, left panel), but necrotic splenocytes significantly increased from 3 to $12 \mathrm{~h}$ pi (Figure 3B, right panel). High percentages of necrotic monocytes/ macrophages particularly at $12 \mathrm{~h}$ pi $(69.6 \%)$ were also detected.

Combining the ELISA values (Figure 1A) with the results of flow cytometry (Figure 3A) in L-78infected mice, a positive correlation between serum proT $\alpha(100-109)$ concentration and the percentage of apoptotic splenic monocytes/macrophages was observed. Before infection both were low, whereas pi the levels of the decapeptide gradually increased following the increase in the percentage of apoptotic cells.

\section{Procaspase-3 was cleaved early during the course of L-78 infection}

To verify activation of the apoptotic pathway during $K$. pneumoniae infection, we determined procaspase- 3 levels in lysates of mouse splenocytes by Western blotting. For controls, we treated HeLa cells with TNF- $\alpha$ and emetine which led the majority of cells to apoptosis, or high concentration of doxorubicin which induced massive necrosis (Supplementary Figure S2). Western blot analysis showed that the levels of procaspase-3 in HeLa lysates were significantly reduced only under apoptotic conditions $(p=0.001)$, and remained unaltered when spleen cells were driven to necrosis (Figure 4).

In our in vivo models, early after infection (3 and 6 h) with L-78, the relative level of procaspase-3 was reduced by half compared to pre-infection levels ( $p=$ 0.0046 and $p=0.0056$, respectively) and was restored to background levels at 12-24 h pi in parallel with bacterial clearance ( $p=0.0407$ at $12 \mathrm{~h} ; p=0.0061$ at $24 \mathrm{~h}$ ). In mice infected with $10^{6} \mathrm{CFU}$ of ATCC 43816, procaspase-3

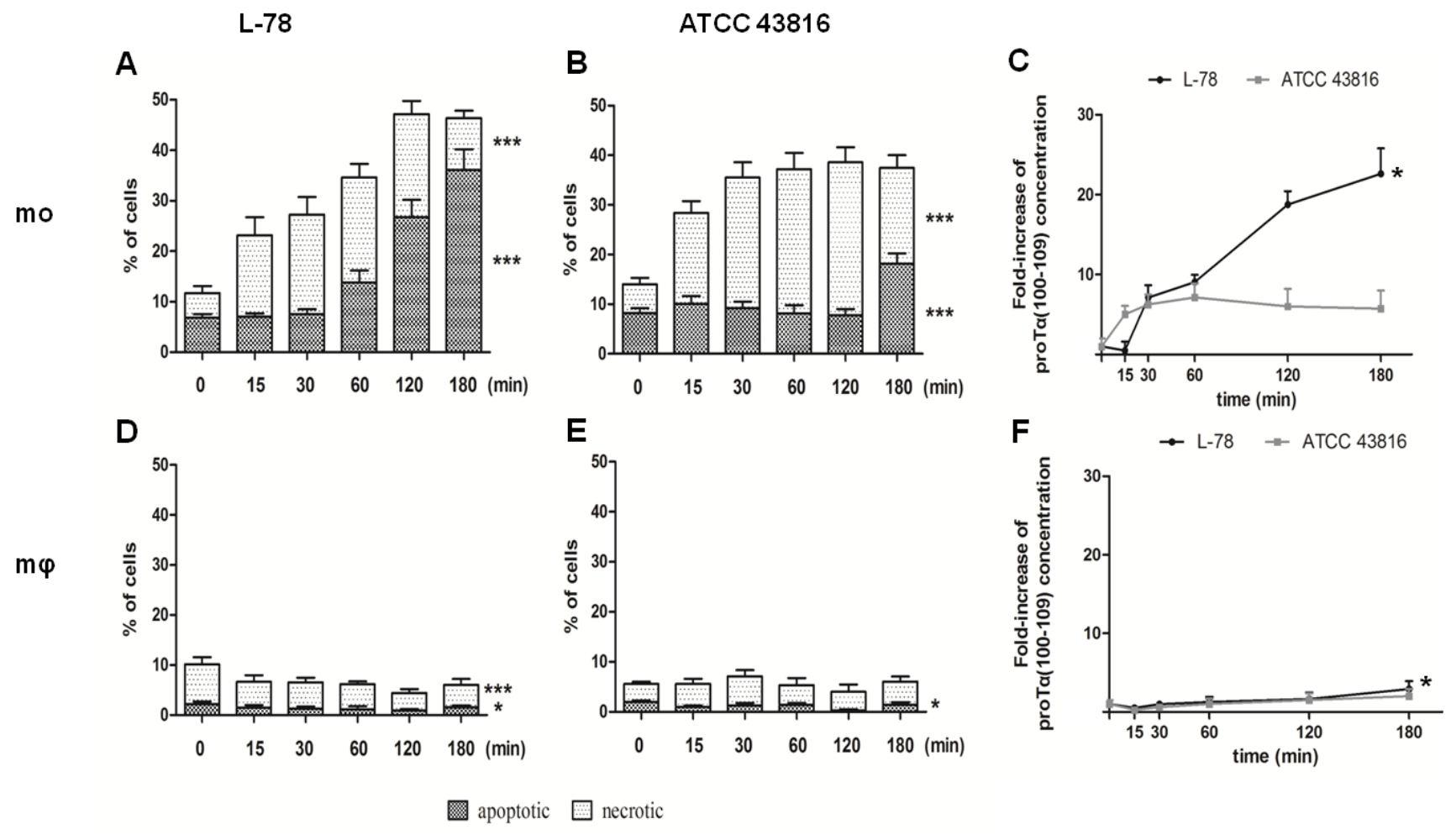

Figure 5: High levels of proTa(100-109) correlated with higher percentages of apoptotic human monocytes in vitro infected with L-78. Human monocytes A. and B. and in vitro-differentiated macrophages D. and E. infected with L-78 A. and D. or ATCC 43816 B. and E. were harvested at 0-180 min, stained with Annexin V/PI and analyzed by flow cytometry. Dark columns show percentages of apoptotic cells; white columns, necrotic cells. Mean values \pm SD from 5 different healthy blood donors are shown. $* * *, p<0.0001$ for L-78-infected apoptotic and necrotic monocytes A., for ATCC 43816-infected apoptotic and necrotic monocytes B. and for L-78-infected necrotic macrophages D.. ${ }^{*}, p=0.0106$ for L-78-infected apoptotic macrophages D. and $p=0.0229$ for ATCC 43816-infected apoptotic macrophages E. (one-way ANOVA). Supernatants from the same cultures were analyzed by ELISA and proT $\alpha(100-109)$ concentration is expressed as mean fold-increase $\pm \mathrm{SD}$ from three independent experiments $\mathbf{C}$. and $\mathbf{F}$. ${ }^{*}, p=0.0015$ for L-78-infected monocytes $\mathbf{C}$. and $p$ $=0.0324$ for L-78-infected macrophage $\mathbf{F}$. (one-way ANOVA). 
protein levels decreased early pi $(p=0.0066$ at $3 \mathrm{~h})$ and progressively increased from 6 to $15 \mathrm{~h}(p=0.0037$ at $6 \mathrm{~h}$; $p=0.0055$ at $12 \mathrm{~h}$ ).

\section{ProTa(100-109) levels increased only in culture supernatants of L-78-infected human monocytes led to apoptosis}

Human monocytes and macrophages co-cultured with L-78 or ATCC 43816 were recovered at 0-180 min and analyzed by flow cytometry, following Annexin V/PI staining. Monocytes infected with L-78 showed a gradual increase in the percentage of apoptotic cells (from $6.85 \%$ at $0 \mathrm{~min}$ to $36.05 \%$ at $180 \mathrm{~min}$ ) (Figure $5 \mathrm{~A}$ ). On the contrary, monocytes infected with ATCC 43816, showed lower percentages of apoptotic cells $(18.15 \%$ at $180 \mathrm{~min})$, but the percentage of necrotic cells increased early pi $(18.30 \%$ at $15 \mathrm{~min})$ and remained high $(30.80 \%$ at $120 \mathrm{~min}$ pi) (Figure 5B). Unlike monocytes, human macrophages resisted infection with both $K$. pneumoniae strains, and much lower percentages of dead cells were recorded $(<$ $10 \%$ ). Specifically, few macrophages incubated with L-78 were apoptotic (1-2\%) (Figure 5D). Macrophages incubated with ATCC 43816 showed a similar pattern of cell death, characterized by few apoptotic $(<2 \%)$ and necrotic $(<5 \%)$ cells (Figure $5 \mathrm{E})$.

In parallel, supernatants of the aforementioned co-cultures were collected and analyzed by our ELISA. Gradually increasing concentrations of proT $\alpha(100-109)$, expressed as fold-increase, paralleled the increase of apoptotic cells from 30 to $180 \mathrm{~min}$ pi in the supernatants of L-78-infected monocytes $(p=0.0015)$. The concentration of proTa(100-109) in the supernatants of ATCC 43816-infected monocytes remained low and relatively constant up to $180 \mathrm{~min}$ pi, as less monocytes were driven to apoptosis (Figure 5C). On the contrary, as macrophages infected with either L-78 or ATCC 43816 remained alive, the quantity of proT $\alpha(100-109)$ detected in their culture supernatant was low (fold-increase $<3$ for both bacterial strains, at all time points) (Figure 5F). Nevertheless, the differences observed in L-78-infected macrophages over time were statistically significant $(p=0.0324)$.

\section{Only L-78 was efficiently phagocytosed by human monocytes and macrophages}

Human monocytes and macrophages were incubated with the two K. pneumoniae strains labeled with 5(6)-carboxyfluorescein diacetate $\mathrm{N}$-succinimidyl ester (CFSE) to visualize and monitor phagocytosis by flow cytometry. Monocytes incubated with L-78 showed a moderate gradual increase in bacterial phagocytosis up to $60 \mathrm{~min}$ pi, which was significantly reduced between 120-180 min. Conversely, monocytes co-incubated with ATCC 43816 for the same time did not phagocytose the bacteria, and minimum internalization was recorded (0.9$5.6 \%$ ) (Figure 6A). In contrast to monocytes, macrophages rapidly and strongly phagocytosed L-78 up to $120 \mathrm{~min}$ pi and only at $180 \mathrm{~min}$ pi, L-78 phagocytosis was reduced by half. A very low percentage of ATCC 43816 was phagocytosed by macrophages ( $<10 \%$ over $180 \mathrm{~min}$ ) (Figure 6B).

Confocal microscopy confirmed the intracellular localization of L-78 in monocytes/macrophages, excluding the possibility of bacterial adherence on the cell surface. Analysis was conducted by examining coverslips on which monocytes and macrophages were co-incubated with CFSE-labeled bacteria for $30 \mathrm{~min}$, a time point where high percentages of phagocytosis were recorded by flow cytometry (Figure 6). In monocytes and especially macrophages infected with L-78, their typical ellipsoidal and round nuclei were surrounded by green fluorescence,
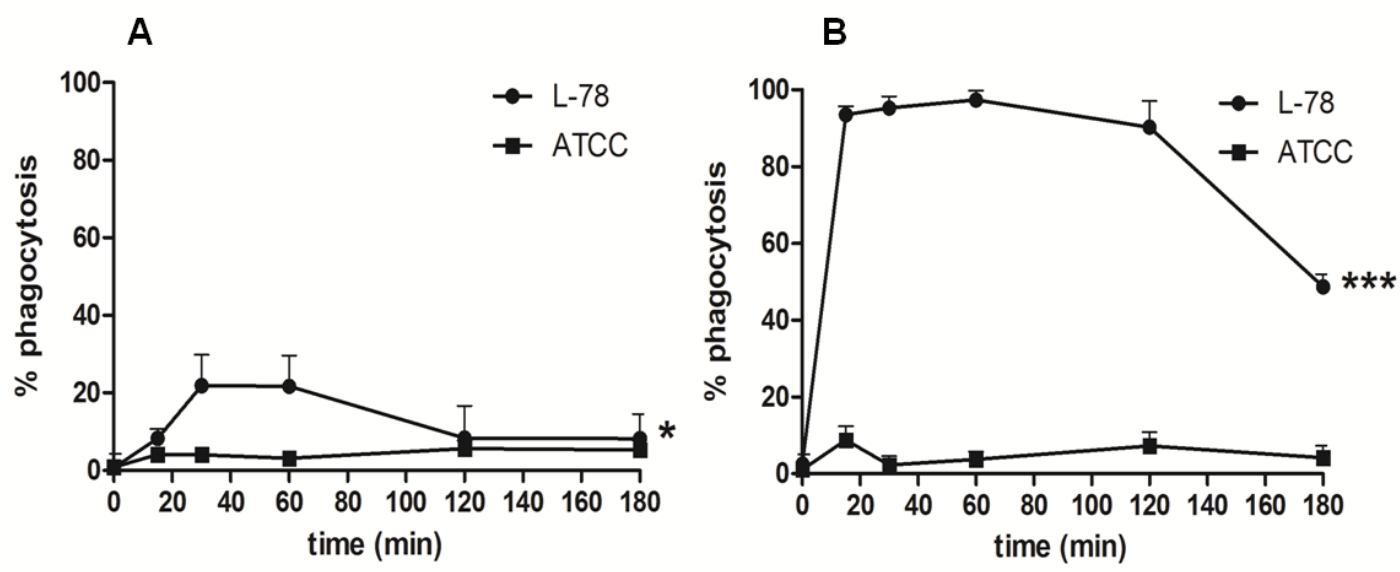

Figure 6: $K$. pneumoniae L-78 was phagocytosed by human macrophages and monocytes. Human monocytes A. and in vitrodifferentiated macrophages B., cultured on coverslips in DMEM supplemented with 1\% FBS without antibiotics, were infected with CFSE-labeled L-78 $(\bullet)$ or ATCC $43816(\bullet)$ for 0-180 min, and analyzed by flow cytometry. Data are means \pm SD from cells derived from 5 different donors. *, $p=0.0266$ for L-78-infected monocytes; ${ }^{* *}, p<0.0001$ for L-78-infected macrophages (one-way ANOVA). 

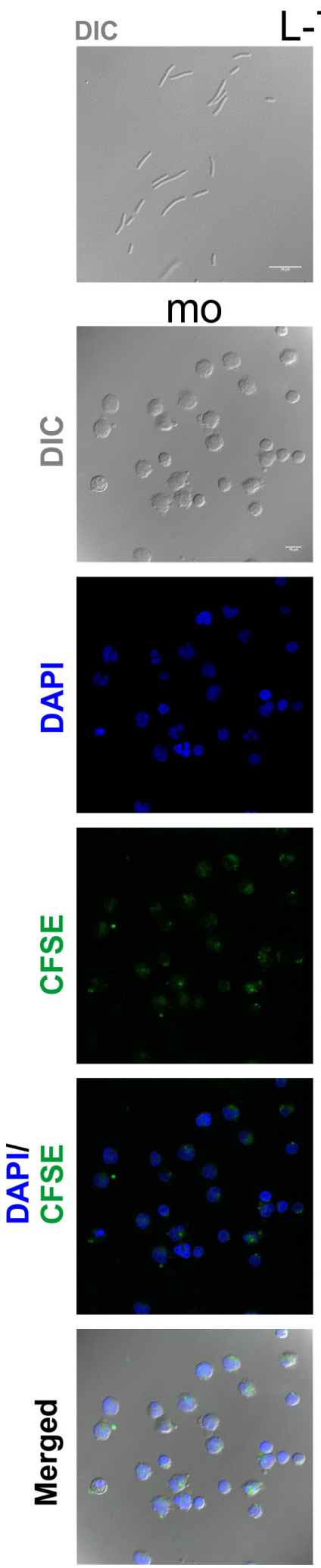
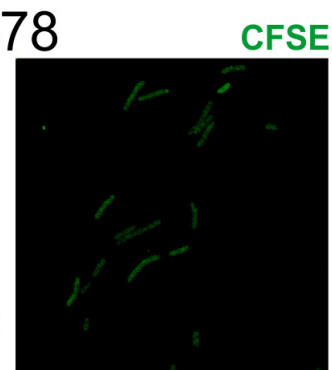

$\mathrm{m} \varphi$
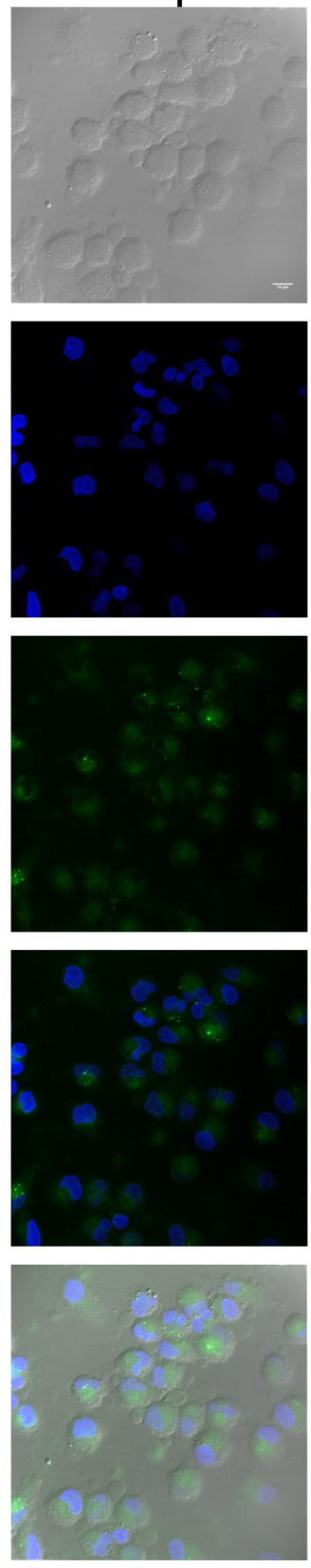

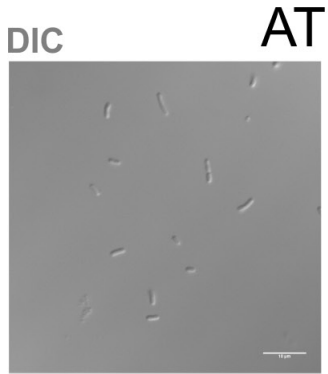

mo
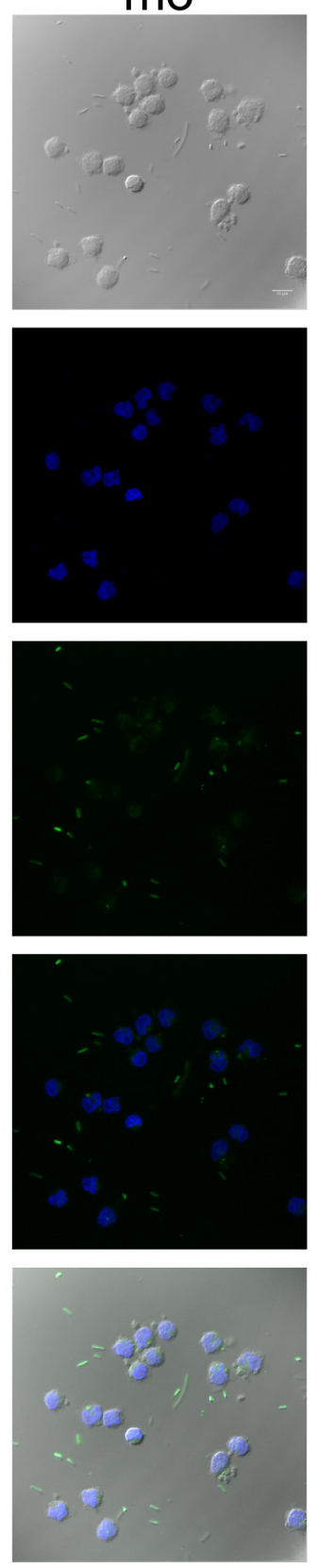

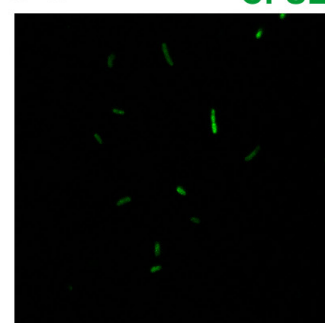

$\mathrm{m} \varphi$
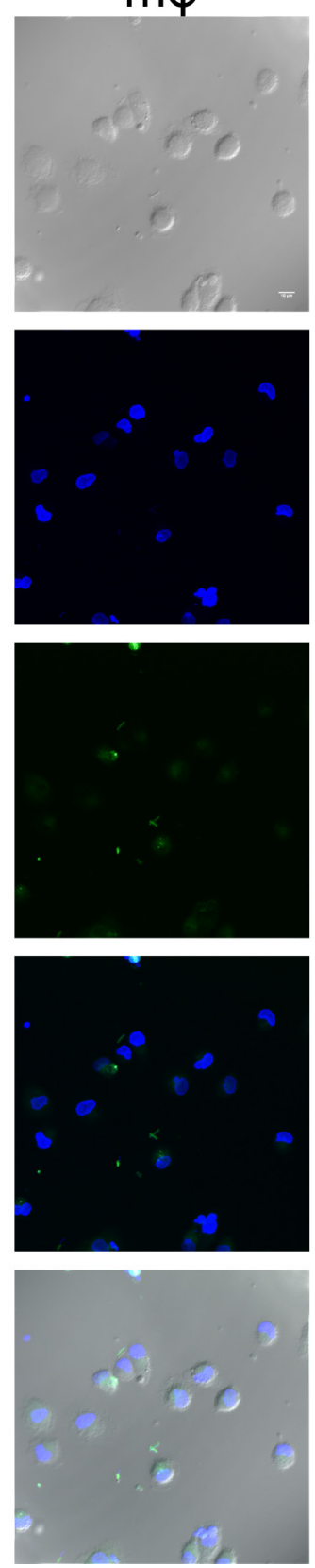

Figure 7: Intracellular localization of $\boldsymbol{K}$. pneumoniae L-78. Cells from the same donors treated as described in legend of Figure 6, were examined using a confocal microscope. ATCC 43816 and L-78 were stained green with CFSE. Nuclei were stained blue with DAPI. The frames show representative optical fields of the labeled bacteria L-78 and ATCC 43816 (first row), and of monocytes (mo) and macrophages $(\mathrm{m} \varphi)$ incubated with L-78 and ATCC 43816 for $30 \mathrm{~min}$. Nomarski images represent the average projection of 6 to 20 optical sections taken at $0.50 \mu \mathrm{m}$ intervals on the $\mathrm{z}$ axis, except the L-78 bacteria (1 optical section). DAPI and CFSE images represent the maximum projection of the same above stacks. Scale bars, $10 \mu \mathrm{m}$. Images from one representative experiment out of 5 performed are shown. 
suggesting that L-78 was internalized and digested by both cell subpopulations. On the contrary, monocytes and macrophages infected with ATCC 43816 emitted blue fluorescence accumulated in their characteristic nuclei, and less green fluorescence, whereas non-phagocytosed bacteria appeared in the extracellular space.

\section{Serum levels of proTa(100-109) can predict mortality due to sepsis early pi}

To validate the significance of our results, we generated a "moderate" model of infection, by infecting mice with $5 \times 10^{3}$ CFU ATCC $43816\left(\mathrm{LD}_{50}\right)$ [13]. In this case $50 \%$ of the animals were expected to die due to sepsis between $30-45 \mathrm{~h}$ pi. We were not able to generate a similar "moderate" mouse model of sepsis using higher inocula of L-78, as bacterial preparations corresponding to $\geq 10^{10}$ $\mathrm{CFU} / \mathrm{mL}$ resulted in difficult to handle highly viscous preparations.

Mice that developed lethal septicemia and died had increased serum concentrations of proT $\alpha(100-109)$ as early as $3 \mathrm{~h}$ pi (Figure $1 \mathrm{C}$, red circles). The differences were more pronounced at 6 and $12 \mathrm{~h} \mathrm{pi}$, where the levels of proT $\alpha(100-109)$ in serum of infected animals that died at $30-45 \mathrm{~h}$ were similar ( $>5 \mathrm{ng} / \mathrm{mL}$ ) to those of mice infected with the high (lethal) inoculum of ATCC 43816 ( $10^{6}$ CFU) (Figure 1B). On the contrary, mice that recovered infection and survived had lower proT $\alpha(100-109)$ levels in their serum at 6 and $12 \mathrm{~h}$ pi, and these resembled more the levels of the decapeptide in the serum of L-78-infected animals ( $<5 \mathrm{ng} / \mathrm{mL}$; Figure 1A).

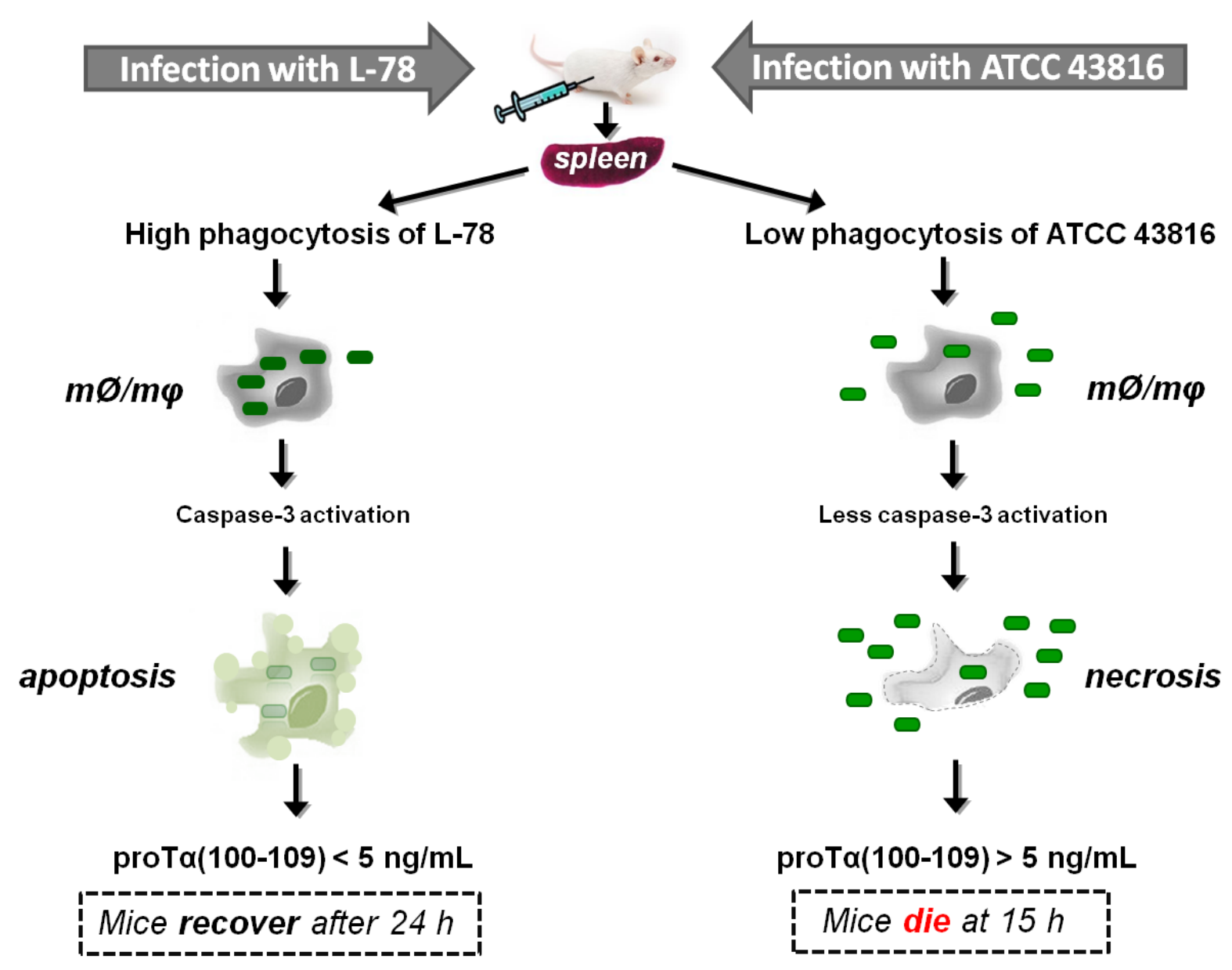

Figure 8: Proposed scenario supporting the eventual use of proTa(100-109) as an early surrogate biomarker of sepsis. Klebsiella pneumoniae strains L-78 (left) and ATCC 43816 (right) intraperitoneally injected in mice enter the bloodstream through the microcirculation of the peritoneum, infect internal organs, e.g. spleen, and multiply. In spleen, monocytes $/ \mathrm{macrophages}(\mathrm{m} \varnothing / \mathrm{m} \varphi)$ phagocytose L-78 (green rectangles) early post-infection $(3 \mathrm{~h})$ and caspase-3 levels are increased, driving cells to apoptosis. In apoptotic cells, proT $\alpha$ is cleaved by activated caspase- 3 and the decapeptide proT $\alpha(100-109)$ is gradually exocytosed. Finally, L-78 is cleared; mice control the infection (at $\sim 24 \mathrm{~h}$ ) and recover. On the contrary, ATCC 43816 is minimally phagocytosed by spleen monocytes/macrophages $(\mathrm{m} \varnothing / \mathrm{m} \varphi)$, thereby remaining in the extracellular space where they rapidly multiply. In this case, monocytes/macrophages are led to necrosis $(12 \mathrm{~h})$ and caspase- 3 is less activated. High levels of DAMPs are extracellularly released, the bacteria are not cleared, sepsis and multiple organ failure occur, and by $15 \mathrm{~h}$ all mice die. 
Overall, 4 infected mice with increased proT $\alpha(100-109)$ concentration in serum between 3-12 h pi died at 30-45 $\mathrm{h}$, suggesting that very early determination of the levels of the decapeptide in their peripheral blood may predict sepsis-induced death.

\section{DISCUSSION}

The necessity of introducing novel biomarkers for prognosis and early prediction of the outcome of septic patients is highly critical. In this study, we used the decapeptide proT $\alpha(100-109)$ as a biomarker to monitor the progression of sepsis in mice infected with $K$. pneumoniae. ProT $\alpha(100-109)$ is the C-terminal fragment of proT $\alpha$, a polypeptide with reported immunostimulatory activity [15], considered a danger signal or alarmin [10, 16-17].

We infected mice with two K. pneumoniae strains: the clinical isolate L-78 and the prototype ATCC 43816. Infection of mice with either strain, increased serum levels of proT $\alpha(100-109)$, but a differential pattern of increase over time was registered. In the L-78 model, the concentration of proT $\alpha(100-109)$ rose in a time-dependent manner after the onset of sepsis. Mice developed visible signs of bacteremia (lethargy, hunched posture, increased breathing rate and shiver), but as L-78 was phagocytosed and presumably successfully cleared, infection was resolved within $48 \mathrm{~h}$. In contrast, in the $10^{6} \mathrm{CFU}$ ATCC 43816 model, the levels of proT $\alpha(100-109)$ significantly increased early pi (at $3 \mathrm{~h}$ ) and remained high until death of all animals at $15 \mathrm{~h}$ pi. In this case, the bacteria rapidly reached the spleen and rose exponentially, as confirmed by spleen cell cultures. These findings are in agreement with previous studies regarding the differential infectivity profiles of the two K. pneumoniae strains [13], as well as with the observed increase in proT $\alpha(100-109)$ serum levels in S. pyogenes-infected mice [11].

To verify that the levels of proT $\alpha(100-109)$ correlate with apoptosis, we assessed the cell death pattern of murine splenocytes following $K$. pneumoniae infection. L-78 infection induced mostly apoptosis, which was more apparent in splenic monocytes/macrophages, while infection with ATCC 43816 induced necrosis, predominantly at later time points pi. Our findings are in agreement with the study of Yang [18], who elegantly demonstrated that in vitro infection of cells with whole live $K$. pneumoniae, induced multiple (apoptotic and necrotic) cell death pathways concurrently. Therefore, we suggest cellular apoptosis and necrosis as causative elements for the presence of proT $\alpha(100-109)$ and probably intact proT $\alpha$ in blood serum of $K$. pneumoniae-infected mice, respectively.

Activation of apoptotic pathways directly relates to alterations in procaspase-3/caspase-3 levels, and results in degradation of cellular components [19], including cleavage of proT $\alpha$ [6]. Indeed, in L-78 infection, the levels of procaspase- 3 were significantly reduced at the early stages pi indicating activation of caspase-3, whereas in the ATCC 43816 model, procaspase-3 levels decreased less. In agreement with the study of Lin [20], who also showed that activated caspase- 3 was significantly elevated in murine liver lysates upon K. pneumoniae infection, our results suggest that L-78 infection massively induces caspase-3-mediated apoptosis at early stages pi in vivo.

To extend the aforementioned host-pathogen interactions in man, we exposed human monocytes/ macrophages to the two K. pneumoniae strains. Monocytes infected with L-78 died mainly by apoptosis, whereas those infected with ATCC 43816 mostly by necrosis. As also shown by others [21], monocytes were more susceptible to death compared to macrophages, which were highly resistant. When we quantified the levels of proT $\alpha(100-109)$ in infected monocyte and macrophage culture supernatants, we recorded significantly elevated proT $\alpha(100-109)$ concentration in supernatants of L-78infected monocytes. In agreement with the in vivo results, high proTa(100-109) levels corresponded to increased percentages of apoptotic cells. Confocal microscopy confirmed that L-78 was mostly internalized early pi, whereas at the same time point, ATCC 43816 was present, almost exclusively, extracellularly. This result is in line with previous data showing that L-78 was efficiently phagocytosed by the murine macrophage cell line J774A.1 [13].

Finally, we tested the predictive ability of our assay in a "moderate" model of sepsis induced by K. pneunoniae ATCC 43816 strain. We infected mice with an inoculum corresponding to the $\mathrm{LD}_{50}$ of ATCC 43816 [13] and quantified serum levels of proT $\alpha(100-109)$ over $48 \mathrm{~h}$. High levels of the decapeptide in the serum of animals which finally died due to sepsis were determined as early as $3 \mathrm{~h}$ pi, suggesting that proT $\alpha(100-109)$ could serve as an early surrogate biomarker for sepsis outcome.

Although experiments in additional infectious models are warranted, to our knowledge this is the first report showing that the levels of proT $\alpha(100-109)$ can serve as a biomarker for monitoring the outcome of bacteria (herein K. pneumoniae)-induced sepsis. Quantification of proT $\alpha(100-109)$ in serum is advantageous compared to HMGB1, thoroughly studied in sepsis. Firstly, generation of proTa(100-109) is a very early apoptotic event (occurring within the first $2 \mathrm{~h}$ ) [6] and so proT $\alpha(100-109)$ can serve as a very early sepsis biomarker, in contrast to HMGB1 that is a late sepsis mediator. Secondly, although the exact mechanism of proT $\alpha(100-109)$ release from cells is yet unknown, the presence of proT $\alpha(100-109)$ extracellularly is strongly associated with massive cell apoptosis induced by an infectious agent. Preliminary results in humans show that serum proT $\alpha(100-109)$ levels increase in septic patients, but not in cases of "sterile inflammation", developed for instance in patients with autoinflammatory diseases (P. Samara et al., unpublished data). In contrast, increased levels of HMGB1 have been 
reported in several non-infectious conditions [22].

Based on the results presented here, we propose a realistic scenario on the significance of extracellular proT $\alpha(100-109)$ quantification relevant to the diverse mechanisms of sepsis induced by the two K. pneumoniae strains (Figure 8): L-78 is phagocytosed by innate immune cells, caspase- 3 is activated and cells are driven to apoptosis. Apoptotic cells gradually excrete proT $\alpha(100-109)$, resulting in progressive stimulation of immune responses. Therefore, no toxicity for instance due to excess cytokine secretion occurs, and this concomitantly with L-78 clearance by monocytes/macrophages, leads to recovery from sepsis. On the contrary, infection with the non-phagocytosed ATCC 43816 leads to massive cell necrosis, abrupt release of high concentrations of alarmins, including proT $\alpha$, excess immune system activation, irreversible septic shock, and death. In any case, determination of proT $\alpha(100-109)$ at the early phase of sepsis suggests its potential utility as an early sepsis biomarker. Clinical studies in humans, currently in progress in our laboratory, will further evaluate the usefulness of serum proT $\alpha(100-109)$ levels as a surrogate biomarker in monitoring bacterial sepsis.

\section{MATERIALS AND METHODS}

\section{Ethical issues}

Experiments involving animals were performed in strict accordance with the guidelines of the European Convention for the Protection of Vertebrate Animals Used for Experimental and Other Scientific Purposes. All animal experiments were approved by the Animal Care and Use Committee of the Hellenic Pasteur Institute and by National Authorities (Veterinary Section of the Greek Republic). The study involving human samples was approved by the Ethical Committee of the $2^{\text {nd }}$ Peripheral Blood Transfusion Unit and Hemophilia Center, "Laikon" General Hospital, Athens, Greece and informed consent was obtained from all subjects. The study was carried out in accordance with the Declaration of Helsinki, as revised in 2013.

\section{Bacterial strains and culture conditions}

Two strains of the Gram-negative bacillus $K$. pneumoniae of different virulence were used. $K$. pneumoniae KPC-Kp ST258 (L-78) is a clinical isolate derived from patients with bacteremia in Greek hospitals during 2009-2011 and its identity has been previously confirmed by standard techniques [13]. K. pneumoniae ATCC 43816 is a K2 strain used in animal models of infection. Both bacterial strains were stored at $-80^{\circ} \mathrm{C}$ in medium containing $20 \%$ glycerol and were grown in freshly prepared Luria-Bertani (LB) agar (SigmaAldrich, St Louis, MO). Bacteria were incubated at $37^{\circ} \mathrm{C}$ in ambient air in order to reach the log phase of growth. Bacterial growth was spectrophotometrically monitored and adjusted to an initial optical density $\left(\mathrm{OD}_{580 \mathrm{~nm}}\right)$ of 0.3 . Prior animal infection, bacterial preparations were adjusted to $10^{9} \mathrm{CFU} / \mathrm{mL}$ for the L-78 strain and $5 \times 10^{4}$ or $10^{7} \mathrm{CFU} / \mathrm{mL}$ for the ATCC 43816 strain.

\section{In silico analysis of $K$. pneumoniae and mouse genomes}

We retrieved $K$. pneumoniae protein sequence data available in publicly accessible sequence databases. In particular, data regarding $K$. pneumoniae complete genomes were retrieved from the NCBI website (http:// www.ncbi.nlm.nih.gov/genome/genomes/815; accessed 9 June 2016). In total, the predicted protein complements for 58 genomes were retrieved. In addition, we retrieved protein sequence sets from the same source for 7 yet incomplete genomes for $K$. pneumoniae strains which are related to the strains studied in this work for completeness. A list of the K. pneumoniae protein datasets used in this work is provided in Supplementary Table S1. Proteins encoded in the mouse genome were downloaded from ENSEMBL (accessed 8 June 2016; build GRCm38.p4) [23]. For representing the proT $\alpha(100-109)$-like peptides, we used the same sequence set and alignments described in [11].

\section{In vivo models of sepsis}

Female ICR (CD-1) mice aged 6 to 8 weeks with a weight of 25-30 g were maintained in the Department of Animal Models for Biomedical Research of the Hellenic Pasteur Institute under pathogen-free conditions. Mice were ip injected with $10^{8} \mathrm{CFU}$ per mouse of the L-78 strain $(n=18), 5 \times 10^{3}$ or $10^{6} \mathrm{CFU} /$ mouse of the ATCC 43816 strain ( $n=10$ and $n=16$, respectively) and monitored every $2 \mathrm{~h}$ for clinical signs of bacteremia (lethargy, hunched posture, increased breathing rate or shiver). Blood was collected by retro-orbital sinus puncture using a glass Pasteur pipette before infection (control, $0 \mathrm{~h}$ ), at $3,6,12,24$, and $48 \mathrm{~h}$ pi for L-78 and $5 \times 10^{3}$ ATCC 43816 groups, and at 3,6,12, and $15 \mathrm{~h}$ pi for $10^{6}$ ATCC 43816 group. At each time point, two animals from the L-78 and $10^{6}$ ATCC 43816 groups were euthanized by cervical dislocation. Spleens were aseptically removed, weighed and homogenized in sterile Dulbecco's phosphate-buffered saline (DPBS; Lonza, Cologne, Germany). An aliquot of the homogenate was analyzed by flow cytometry and Western blotting, while the remaining homogenate was diluted and plated onto LB agar to determine the number of viable microorganism CFUs. 


\section{Isolation of peripheral blood mononuclear cells (PBMCs)}

Human buffy coat preparations were used as a source of peripheral blood mononuclear cells (PBMCs) isolated by centrifugation over Ficoll-Histopaque (SigmaAldrich) density gradient. PBMCs were resuspended (10$15 \times 10^{6} / \mathrm{mL}$ ) in DMEM, supplemented with $10 \%$ heatinactivated fetal bovine serum (FBS), $10 \mathrm{mM}$ Hepes, $5 \mu \mathrm{g} /$ $\mathrm{mL}$ gentamycin and $10^{2} \mathrm{U} / \mathrm{mL}$ penicillin/streptomycin (all from Lonza). Highly enriched monocytes ( $>85 \% \mathrm{CD} 14+$ as verified by flow cytometry following staining with FITC anti-human CD14 antibody [BioLegend, London, UK]) were obtained from PBMCs by plastic adherence for $2 \mathrm{~h}$ at $37^{\circ} \mathrm{C}$ in a humidified $5 \% \mathrm{CO}_{2}$ incubator. Macrophages were differentiated from monocytes by incubation with $100 \mathrm{ng} / \mathrm{mL}$ recombinant human granulocyte macrophage colony-stimulating factor (R\&D Systems $\mathrm{GmbH}$, Wiesbaden-Nordenstadt, Germany) for 5 days [24]. Differentiation was confirmed by flow cytometry, by staining macrophages with APC-Cy7 anti-human CD206 antibody (BioLegend). Both monocytes and macrophages were in vitro infected with L-78 or ATCC 43816. The levels of proT $\alpha(100-109)$ were quantified in supernatants by ELISA, whereas the type of induced cell death and their phagocytic ability were analyzed by flow cytometry and confocal microscopy.

\section{Enzyme-linked immunosorbent assay (ELISA) for proTa(100-109)}

Blood samples from each mouse were individually treated as previously described [11]. Precipitated mouse sera or supernatants from K. pneumoniae-infected human monocytes or macrophages were analyzed in duplicates by our in-house developed ELISA, and the quantity of proT $\alpha(100-109)$ was expressed in $\mathrm{ng} / \mathrm{mL}$ using the linear part of the standard curve [11].

\section{Evaluation of cell apoptosis}

To evaluate morphological changes in splenocytes of Klebsiella-infected mice, as well as in Klebsiellainfected human monocytes/macrophages, cells were stained with Annexin V and PI [25]. Annexin V binds to phosphatidylserine exposed on the outer leaflet of the cell membrane of apoptotic cells, while PI is a DNAbinding dye, which enters cells when their membranes are ruptured. For cell labeling, the FITC Annexin V apoptosis kit with PI (BioLegend) was used and staining was performed as recommended by the manufacturer. For adherent cells, i.e., human monocytes and macrophages, detachment via $1 \mathrm{X}$ trypsin/EDTA solution (Lonza) was used and viability was $>90 \%$ as confirmed by Trypan
Blue exclusion [26]. Cells were washed twice with PBS and resuspended in Annexin V binding buffer at a concentration of $10^{6}$ cells $/ \mathrm{mL}$. One hundred $\mu \mathrm{L}$ of cell suspension were transferred in FACS tubes, where $5 \mu \mathrm{L}$ of FITC Annexin V and $10 \mu \mathrm{L}$ of PI were added. Cells were gently vortexed and incubated for $15 \mathrm{~min}$ at room temperature in the dark. Four hundred $\mu \mathrm{L}$ of Annexin V binding buffer were added to each tube and cells were analyzed by a FACSCanto II flow cytometer (BectonDickinson [BD] Biosciences, Erembodegem, Belgium) equipped with FACSDiva software (BD Biosciences). Monocytes/macrophages from mouse spleens were gated using phycoerythrin anti-mouse CD11b antibody (BioLegend) and analyzed separately.

\section{Western blotting}

Splenocytes were lysed in lysis buffer $(50 \mathrm{mM}$ Tris-HCl, $150 \mathrm{mM} \mathrm{NaCl}, 2 \mathrm{mM}$ EDTA, $\mathrm{pH} 7.6,1 \%$ Triton X-100 [v/v]) supplemented with Complete ${ }^{\circledR}$ protease inhibitor cocktail (Roche Life Science, Manheim, Germany) for $30 \mathrm{~min}$ on ice. Cell lysates were centrifuged at $10,000 \mathrm{~g}$ for $10 \mathrm{~min}$ at $4^{\circ} \mathrm{C}$ and protein concentration in the supernatants was determined using the Bradford protein assay (SERVA Electrophoresis GmbH, Heidelberg, Germany). In parallel, HeLa cells (ATCC CCL-2), led in vitro to apoptosis (4 $\mathrm{h}$ incubation with $5 \mathrm{ng} / \mathrm{mL}$ TNF- $\alpha$ and $1 \mu \mathrm{g} / \mathrm{mL}$ emetine [both from Sigma-Aldrich]) or necrosis ( $4 \mathrm{~h}$ incubation with $100 \mu \mathrm{g} /$ $\mathrm{mL}$ doxorubicin [Sigma-Aldrich]), were used as controls [6]. Equal amounts of protein $(20 \mu \mathrm{g} / \mathrm{lane})$ were separated by SDS-PAGE on $15 \%(\mathrm{w} / \mathrm{v})$ gels and transferred onto nitrocellulose membranes. The blots were blocked in a solution of 5\% (w/v) nonfat dry milk in Tris-buffered saline containing Tween-20 (TBS-T; $20 \mathrm{mM}$ Tris-HCl, $\mathrm{pH} 7.5,137 \mathrm{mM} \mathrm{NaCl}, 0.05 \%$ [v/v] Tween-20) for $1 \mathrm{~h}$ at room temperature, followed by overnight incubation at $4^{\circ} \mathrm{C}$ with primary antibodies against procaspase-3 (1:500 in TBS-T; ab44976, Abcam, Cambridge, UK) or $\beta$-actin (1:2500; Sigma-Aldrich). After washing with TBS-T (4x5 min), blots were incubated with the appropriate HRP-conjugated secondary antibody (1:5000 in TBS-T containing $1 \%[\mathrm{w} / \mathrm{v}]$ nonfat dry milk) for $1 \mathrm{~h}$ at room temperature, washed again with TBS-T $(4 \times 5 \mathrm{~min})$, developed using the enhanced chemiluminescence reagent (ECL reagent; Amersham Biosciences, Buckinghamshire, UK) and quantified by densitometry (Gel Analyzer v.1.0, Biosure, Athens, Greece). All densitometric values were normalized against $\beta$-actin levels. Procaspase- 3 levels in control samples were set at one, and the respective values of treated samples are expressed as fold-induction over control samples. 


\section{Analysis of phagocytosis by flow cytometry}

The two strains of $K$. pneumoniae were labeled with the fluorescent dye CFSE (Sigma-Aldrich) according to Sokolovska [27]. Human monocytes or macrophages were incubated with the bacteria following the protocol described in [27] with slight modifications. In brief, $4.5-5 \times 10^{5}$ monocytes or macrophages were plated per well of a 6-well culture plate (Greiner Bio-One $\mathrm{GmbH}$, Frickenhausen, Germany) in $2 \mathrm{~mL}$ DMEM-1\% FBSwithout antibiotics (w/a). The medium was replaced with $1.5 \mathrm{~mL}$ ice-cold DMEM-1\% FBS-w/a containing the CFSE-labeled bacterial particles at a multiplicity of infection (MOI) of 25. Plates were centrifuged $(100 \mathrm{~g}$, $4^{\circ} \mathrm{C}, 5 \mathrm{~min}$ ) to allow bacteria to settle onto the cells and incubated at $37^{\circ} \mathrm{C}$ for $15,30,60,120$, and $180 \mathrm{~min}$. Wells placed on ice $\left(4^{\circ} \mathrm{C}\right)$ were used as controls. Phagocytosis was stopped by adding $1.5 \mathrm{~mL}$ of ice-cold DPBS containing $5 \mathrm{mM}$ EDTA (Lonza) to each well. Cells were washed twice with DPBS/EDTA, once with DPBS and were detached using $1 \mathrm{X}$ trypsin/EDTA solution. Cells were then centrifuged, resuspended in $400 \mu \mathrm{L}$ DPBS, transferred to FACS tubes placed on ice, and immediately analyzed by flow cytometry. The results are presented as percentage of monocytes or macrophages bearing internalized bacteria (\% phagocytosis) versus time.

\section{Analysis of phagocytosis by confocal microscopy}

Human monocytes or macrophages were grown on sterilized 12x12 mm coverslips (Sigma-Aldrich) precoated with poly-L-lysine $\left(50 \mu \mathrm{g} / \mathrm{mL}\right.$ for $1 \mathrm{~h}$ at $37^{\circ} \mathrm{C}$; SigmaAldrich) and placed in 6-well culture plates at $37^{\circ} \mathrm{C}$ in a humidified 5\% $\mathrm{CO}_{2}$ incubator. Cells were infected with the two Klebsiella strains for $30 \mathrm{~min}$, as described in the previous paragraph, fixed with $4 \%$ paraformaldehyde diluted in DPBS for $15 \mathrm{~min}$ at room temperature and washed three times with DPBS. Each coverslip was placed on a single slide containing mounting medium with DAPI (Sigma-Aldrich) and protected from light. Specimens were examined on a multiphoton confocal microscope Leica TCS SP8 MP (Wetzlar, Germany) equipped with an Argon laser (excitation lines at 458, 476, 488, 496, and 514nm) and an IR MaiTai DeepSee Ti:Sapphire laser (Spectra-Physics, Santa Clara, CA, USA) for multiphoton applications. Images were acquired with the spectral detector of the microscope using appropriate emission wavelength ranges; CFSE (green) at $500-550 \mathrm{~nm}$ and DAPI (blue) at $420-500 \mathrm{~nm}$. Acquisition was performed with the LAS X software (Leica Microsystems CMS $\mathrm{GmbH}$, Wetzlar, Germany) using the same parameters (laser power, gain, pinhole, speed and analysis) for all specimens. Images were acquired as stacks of 6 to 20 optical sections with a Z-step of $0.50 \mu \mathrm{m}$. Average or Maximum Projections of the stacks were created with the LAS X software and minor adjustments to contrast, brightness and levels with Adobe Photoshop CC (Adobe Systems Inc., San Jose, CA, USA) for better representation.

\section{Statistical analysis}

Each in vitro and in vivo experiment was conducted at least three times. All data were analyzed using GraphPad Prism 5 software (GraphPad Software, San Diego, CA, USA). Results are expressed as means \pm standard deviation (SD). For statistics, we used Student's $t$-test and one-way analysis of variance (ANOVA) followed, where indicated, by Dunnett's test. $P$-values $<$ 0.05 were considered statistically significant.

\section{Abbreviations}

CFU, colony-forming unit; DAMP, damageassociated molecular pattern; HMGB1, high-mobility group box 1 protein; $K$. pneumoniae, Klebsiella pneumoniae; PI, propidium iodide; proT $\alpha$, prothymosin alpha; S. pyogenes, Streptococcus pyogenes.

\section{Author contributions}

P.S.: performed the experiments, analyzed data and wrote the manuscript. V.M.: coordinated bacterial cultures, mouse experiments, drafted and reviewed the manuscript. M.Z. and S.G.D.: helped in the microscopy experiments. O.M. and P.P.: helped in Western Blots. V.J.P.: performed the in silico analysis. H.K. and W.V.: helped in the ELISA experiments. O.T.: designed and coordinated the whole study, drafted and reviewed the manuscript.

\section{ACKNOWLEDGMENTS}

We thank Prof. Spyros Efthimiopoulos for providing the anti-procaspase-3 antibody, Prof. Efstratios Valakos for statistical analysis, Dr. Ioannis Voutsas for flow cytometry analysis and Georgios Miliotis for his assistance in bacterial preparations.

\section{CONFLICTS OF INTEREST}

No conflict of interest is disclosed.

\section{FUNDING}

This work was partly supported by IKY Fellowships of Excellence for Postgraduate Studies in Greece-Siemens Program to PS, NATO Science for Peace and Security Programme (SfP 982838) to WV and OT, and IKYDA $264 / 2016$ to HK and OT. The funders had no role in study 
design, data collection and interpretation, or the decision to submit the work for publication.

\section{REFERENCES}

1. Lakshmikanth CL, Jacob SP, Chaithra VH, de Castro-FariaNeto HC, Marathe GK. Sepsis: in search of cure. Inflamm Res. 2016; 65: 587-602. doi: 10.1007/s00011-016-0937-y.

2. Biron BM, Ayala A, Lomas-Neira JL. Biomarkers for sepsis: what is and what might be? Biomark Insights. 2015; 10: 7-17. doi: 10.4137/BMI.S29519.

3. Fontaine M, Lepape A, Piriou V, Venet F, Friggeri A. Innate danger signals in acute injury: From bench to bedside. Anaesth Crit Care Pain Med. 2016; 35: 283-92. doi: 10.1016/j.accpm.2015.10.009.

4. Fink MP. Animal models of sepsis. Virulence. 2014; 5: 14353. doi: 10.4161/viru.26083.

5. Samara P, Ioannou K, Tsitsilonis OE. Prothymosin alpha and immune responses: are we close to potential clinical applications? Vit Horm. 2016; 102: 179-207. doi: 10.1016/ bs.vh.2016.04.008.

6. Evstafieva AG, Belov GA, Rubtsov YP, Kalkum M, Joseph B, Chichkova NV, Sukhacheva EA, Bogdanov AA, Pettersson RF, Agol VI, Vartapetian AB. Apoptosis-related fragmentation, translocation, and properties of human prothymosin alpha. Exp Cell Res. 2003; 284: 211-23.

7. Mosoian A, Teixeira A, Burns CS, Sander LE, Gusella GL, He C, Blander JM, Klotman P, Klotman ME. Prothymosinalpha inhibits HIV-1 via Toll-like receptor 4-mediated type I interferon induction. Proc Natl Acad Sci USA. 2010; 107: 10178-83. doi: 10.1073/pnas.0914870107.

8. Ioannou K, Derhovanessian E, Tsakiri E, Samara P, Kalbacher H, Voelter W, Trougakos IP, Pawelec G, Tsitsilonis OE. Prothymosin $\alpha$ and a prothymosin $\alpha$-derived peptide enhance $\mathrm{T}(\mathrm{H}) 1$-type immune responses against defined HER-2/neu epitopes. BMC Immunol. 2013; 14: 43 56. doi: 10.1186/1471-2172-14-43.

9. Omotuyi O, Matsunaga H, Ueda H. Evidence for ProT $\alpha$ TLR4/MD-2 binding: molecular dynamics and gravimetric assay studies. Expert Opin Biol Ther. 2015; 15: S223-9. doi: 10.1517/14712598.2015.1005597.

10. Ioannou K, Samara P, Livaniou E, Derhovanessian E, Tsitsilonis OE. Prothymosin alpha: a ubiquitous polypeptide with potential use in cancer diagnosis and therapy. Cancer Immunol Immunother. 2012; 61: 599-614. doi: 10.1007/ s00262-012-1222-8.

11. Samara P, Kalbacher H, Ioannou K, Radu DL, Livaniou E, Promponas VJ, Voelter W, Tsitsilonis O. Development of an ELISA for the quantification of the C-terminal decapeptide prothymosin $\alpha(100-109)$ in sera of mice infected with bacteria. J Immunol Methods. 2013; 395: 5462. doi: 10.1016/j.jim.2013.06.011.

12. Pitout JD, Nordmann P, Poirel L. Carbapenemaseproducing Klebsiella pneumoniae, a key pathogen set for global nosocomial dominance. Antimicrob Agents Chemother. 2015; 59: 5873-84. doi: 10.1128/AAC.0101915.

13. Tzouvelekis LS, Miriagou V, Kotsakis SD, Spyridopoulou K, Athanasiou E, Karagouni E, Tzelepi E, Daikos GL. KPC-producing, multidrug-resistant Klebsiella pneumoniae sequence type 258 as a typical opportunistic pathogen. Antimicrob Agents Chemother. 2013; 57: 5144-6. doi: 10.1128/AAC.01052-13.

14. Franklin GA, Scott MJ, Patel M, Hoth JJ, Peyton JC, Cheadle WG. A novel model of pneumonia from intraperitoneal injection of bacteria. Am J Surg. 2003; 186: 493-9.

15. Samara $\mathrm{P}$, Ioannou $\mathrm{K}$, Neagu M, Arnogiannaki N, Ardavanis A, Voelter W, Tsitsilonis O. The C-terminal decapeptide of prothymosin $\alpha$ is responsible for its stimulatory effect on the functions of human neutrophils in vitro. Int Immunopharmacol. 2013; 15: 50-7. doi: 10.1016/j.intimp.2012.11.011.

16. Bianchi ME. DAMPs, PAMPs and alarmins: all we need to know about danger. J Leukoc Biol. 2007; 81: 1-5. doi: 10.1189/jlb.0306164.

17. Theoharides TC. Danger signals and inflammation. Clin Ther. 2016; 38: 996-9. doi: 10.1016/j.clinthera.2016.03.036.

18. Yang PY, Chen WX, Chang FY, Chen HW, Lin CH, Hsu YC, Wu JH. HepG2 cells infected with Klebsiella pneumoniae show DNA laddering at apoptotic and necrotic stages. Apoptosis. 2012; 17: 154-63. doi: 10.1007/s10495011-0666-1.

19. Nicholson DW. Caspase structure, proteolytic substrates, and function during apoptotic cell death. Cell Death Differ. 1999; 6: 1028-42.

20. Lin YC, Lu MC, Lin C, Chiang MK, Jan MS, Tang HL, Liu HC, Lin WL, Huang CY, Chen CM, Lai YC. Activation of IFN- $\gamma / \mathrm{STAT} / \mathrm{IRF}-1$ in hepatic responses to Klebsiella pneumoniae infection. PLoS One. 2013; 8: e79961. doi: 10.1371/journal.pone.0079961.

21. Webster SJ, Daigneault M, Bewley MA, Preston JA, Marriott HM, Walmsley SR, Read RC, Whyte MK, Dockrell DH. Distinct cell death programs in monocytes regulate innate responses following challenge with common causes of invasive bacterial disease. J Immunol. 2010; 185: 2968-79. doi: 10.4049/jimmunol.1000805.

22. Andersson U, Tracey K. HMGB1 is a therapeutic target for sterile inflammation and infection. Annu Rev Immunol. 2011; 29: 139-62. doi: 10.1146/annurevimmunol-030409-101323.

23. Flicek P, Amode MR, Barrell D, Beal K, Brent S, CarvalhoSilva D, Clapham P, Coates G, Fairley S, Fitzgerald S, Gil L, Gordon L, Hendrix M, et al. Ensembl 2012. Nucleic Acids Res. 2012; 40: D84-90. doi: 10.1093/nar/gkr991.

24. Rey-Giraud F, Hafner M, Ries $\mathrm{CH}$. In vitro generation of monocyte-derived macrophages under serum-free conditions improves their tumor promoting functions. PLoS 
One. 2012; 7: e42656. doi: 10.1371/journal.pone.0042656.

25. Bauer M, Goldstein M, Christmann M, Becker H, Heylmann D, Kaina B. Human monocytes are severely impaired in base and DNA double-strand break repair that renders them vulnerable to oxidative stress. Proc Natl Acad Sci USA. 2011; 108: 21105-10. doi: 10.1073/pnas.1111919109.

26. Jin X, Kruth HS. Culture of macrophage colony-stimulating factor differentiated human monocyte-derived macrophages. J Vis Exp. 2016; 112: e54244. doi: 10.3791/54244.
27. Sokolovska A, Becker CE, Stuart LM. Measurement of phagocytosis, phagosome acidification, and intracellular killing of Staphylococcus aureus. Curr Protoc Immunol. 2012; Chapter 14:Unit14.30. doi: 10.1002/0471142735. im1430s99. 